\title{
Cavity cooling a trapped nanosphere in vacuum
}

P. F. Barker, J. Millen, P. G. Z. Fonseca, T. Mavrogordatos, T. S. Monteiro

P. F. Barker, J. Millen, P. G. Z. Fonseca, T. Mavrogordatos, T. S. Monteiro, "Cavity cooling a trapped nanosphere in vacuum," Proc. SPIE 9164, Optical Trapping and Optical Micromanipulation XI, 916403 (16 September 2014); doi: $10.1117 / 12.2064393$

SPIE Event: SPIE NanoScience + Engineering, 2014, San Diego, California, United States 


\title{
Cavity cooling a trapped nanosphere in vacuum
}

\author{
P. F. Barker, J. Millen, P. G. Z. Fonseca, T. Mavrogordatos and T. S. Monteiro \\ Dept of Physics and Astronomy, University College London, Gower Street, London, United \\ Kingdom;
}

\begin{abstract}
We describe recent experiments that have demonstrated cavity optomechanical cooling of the center-of-mass motion of nanospheres. The naturally charged silica spheres are levitated within vacuum using an overlapping optical and electrodynamical trap. Using this system we have cavity cooled trapped nano-spheres of radius $200 \mathrm{~nm}$ from above room temperature to less than $10 \mathrm{~K}$ in a vacuum of $10^{-4} \mathrm{mbar}$.
\end{abstract}

Keywords: Laser cooling, optical trapping

\section{INTRODUCTION}

There is currently significant interest in utilising optically trapped nano- and microspheres in vacuum as highly undamped oscillators for exploring quantum mechanics in a new mass regime ${ }^{1}$. Masses ranging from $10^{-17}-$ $10^{-9} \mathrm{~kg}$ can be trapped, offering an ideal test-bed for exploring wavefunction collapse models and decoherence at these large mass scales ${ }^{2}$. Particles trapped and cooled in vacuum, may act as highly sensitive force sensors, and are seen as ideal candidates for exploring extensions to classical Newtonian gravity on small length scales and for high frequency gravitational wave detection ${ }^{3}$. There has been considerable success in cooling nano- and micromechanical oscillators, but as they are typically connected to a thermal bath the achievable mechanical Q-factor is limited ${ }^{4}$. Levitation reduces mechanical damping and decoherence mechanisms, with mechanical Q-factors as high as $10^{12}$ predicted when operated in high vacuum ${ }^{11}$. In addition, the mechanical frequency of a levitated particle can be tuned via the trap depth, and the trap can be turned off allowing measurements in a field free, non-perturbed state.

An important step towards utilising these particles is the development of methods that can efficiently cool them. Cavity cooling, which has been developed for any polarisable particle, does not require a detailed knowledge of the internal electronic structure ${ }^{5}$. Nanoparticles interact strongly with an optical cavity field via their polarisability, and cooling occurs when the input field is red detuned from resonance. In this process, the cavity damps the motion of the trapped sphere by preferentially scattering blue-shifted photons out of the cavity. Cavity cooling of nanoparticles has been demonstrated recently for silicon nanoparticles traveling through a cavity ${ }^{6}$, and for particles trapped in the cavity in the presence of a damping buffer gas at approximately $1 \mathrm{mbar}^{7}$. Operation while trapped in vacuum has not been demonstrated as both parametric heating and internal heating (via small absorption by the trapped particle of trapping light) leads to ejection or loss of the particles from an optical trap ${ }^{8,9}$. In this paper we describe nanoparticle cavity cooling experiments in which we use a Paul trap to electrodynamically levitate charged silica nanospheres while the field from a medium finesse optical cavity further confines and cools them.

\section{CAVITY COOLING}

Cavity cooling has been demonstrated experimentally for atoms and ions, but only recently has the cooling of nanoparticles using this method been studied ${ }^{10-14}$. In these schemes a single nanoscale particle is trapped within an optical cavity. Typically one optical field is used for trapping while another optical field is used for cooling ${ }^{11,13,14}$. Cooling occurs when the input field is red-detuned from the optical cavity resonance, and the center-of-mass mechanical motion is damped by scattering blue-shifted light out of the cavity. This damping process can also be understood in a time-dependent picture by considering a polarisable particle moving through

Further author information: (Send correspondence to P.F.B.)

P.F.B.: E-mail: p.barker@ucl.ac.uk, Telephone: +44 (0)207 6797331

Optical Trapping and Optical Micromanipulation XI, edited by Kishan Dholakia,

Gabriel C. Spalding, Proc. of SPIE Vol. 9164, 916403 - ( 2014 SPIE

CCC code: $0277-786 X / 14 / \$ 18 \cdot$ doi: $10.1117 / 12.2064393$

Proc. of SPIE Vol. $9164916403-1$ 


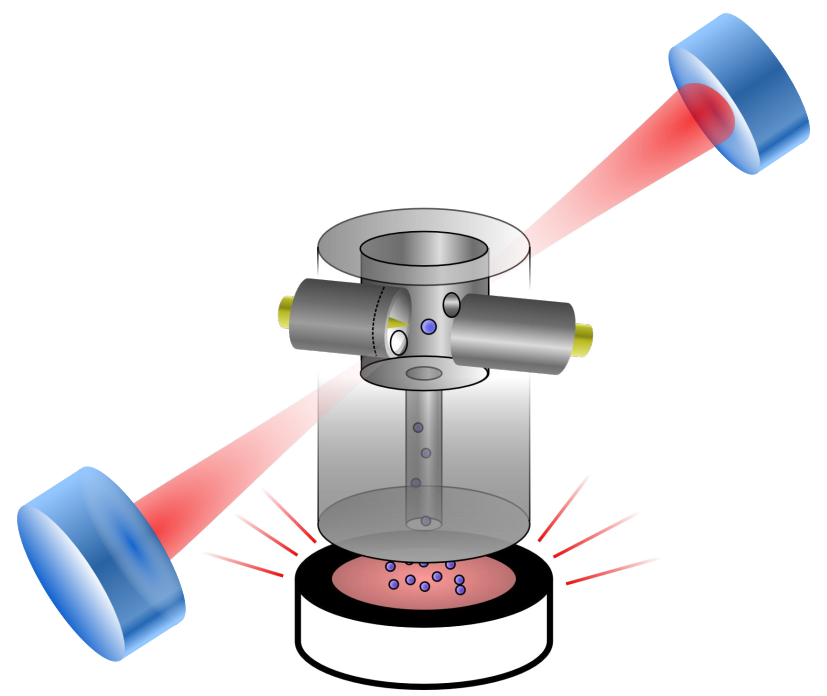

Figure 1. The hybrid trap used for cooling the centre-of-mass motion of a levitated silica nanosphere. A intracavity optical field passes through the centre of the Paul trap producing an overlapping optical and electrical potential suitable for cavity cooling in vacuum.

the periodic, standing-wave field of a cavity. When the particle moves through the standing-wave field, there is a slight increase in the optical path length in the cavity and a decrease in its resonant frequency. For an input field that is red-detuned from the cavity resonance, any decrease in the cavity resonance frequency brings the input field closer to resonance, acting to increase the intracavity intensity and thus also the dipole force on the particle. The finite time required for the intracavity intensity to increase in a high-finesse cavity means that the particle passing through an antinode in the cavity loses more energy than it gains. This dissipation process applies to both trapped and untrapped particles. Such a scheme only relies on the optical path length change induced by a polarisable particle in the cavity. Since all particles are to some extent polarisable, the scheme is amenable to complex massive particles such as nanosheres.

Fast and efficient cavity cooling has been shown to occur when the particle is located in the largest gradient near an anti-node of the standing-wave field of the cavity. To locate the particle in the position of maximum cooling an additional optical field can be used. This could be an optical tweezers ${ }^{10}$ or another cavity field ${ }^{7,11,13,14}$. However, high intensity light is needed for trapping and cooling, and even small absorption of light can lead to heating which can then be transferred via collisions with the surrounding gas to the centre-of-mass of the particle $^{8}$. Thus, without additional damping ${ }^{15}$, to date it has been impossible to trap in vacuum. A Paul trap is an attractive means for levitation of nanoparticles in vacuum because, unlike in optical traps, there is no internal heating. Additionally, the trap can be considerably deeper than an optical trap and is therefore less susceptible to parametric heating processes. Trap frequencies are, however, low (in the $100 \mathrm{~Hz}$ range) and therefore this type of levitation alone is not a suitable candidate for cooling to the quantum regime. We demonstrate a hybrid trap which utilises a Paul trap for levitation and an optical cavity field for additional confinement (higher trap frequencies) and cooling. This is an attractive alternative to the all-optical cooling schemes considered so far and is a promising means to achieve ground-state cooling.

\section{COOLING TRAPPED NANOPARTICLES}

We consider cavity cooling in the hybrid electro-optical trap shown in Fig. 1. Here a silica nanosphere, typically with a 1-3 elementary charges on the surface, is trapped within a Paul trap. Our trap consists of two opposing cylindrical electrodes of diameter $1 \mathrm{~mm}$ on which a high voltage at audio frequencies is applied. The electrodes, when combined with concentric grounded shield electrodes, provide a well defined, near quadrupole trap with excellent optical access. As shown in the figure the intracavity optical field passes through the centre of the Paul trap producing an overlapping optical and electrical potential suitable for cavity cooling. A diagram showing the 


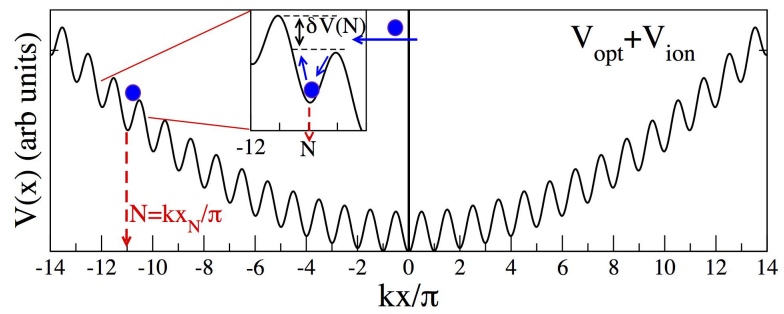

Figure 2. A diagram showing the hybrid potential created the overlapping optical and electrodynamic potentials due to the cavity field and the Paul trap. The nanosphere can be trapped within an optical antinode at position N.

hybrid potential created these two overlapping potentials is shown in Fig. 2. Here the deep, but spatially broad, potential of the Paul trap is modulated by the periodic potential of the standing-wave optical field. Cooling occurs when the nanoparticle has low enough energy to be trapped and localised within a single antinode of the standing wave.

The equation of motion for the amplitude $a$ of the optical cavity field is:

$$
\dot{a}(t)=i \Delta a-i E+i A a e^{-2\left(z^{2}+y^{2}\right) / w_{0}^{2}} \cos ^{2} k x-\frac{\kappa}{2} a,
$$

and the nanoparticle momentum along the direction of the field (x-direction) is given by:

$$
\ddot{x}(t)=-\frac{\hbar k A}{m}|a(t)|^{2} e^{-2\left(z^{2}+y^{2}\right) / w_{0}^{2}} \sin 2 k x-\Gamma_{M} \dot{x}-\omega_{T}^{2} x \cos \left(\omega_{d} t\right) .
$$

The momentum perpendicular to the field is given by:

$$
\ddot{y}(t)=-\frac{4 y}{m w_{0}^{2}} \hbar A|a(t)|^{2} e^{-2\left(z^{2}+y^{2}\right) / w_{0}^{2}} \cos ^{2} k x-\Gamma_{M} \dot{y}-\omega_{T}^{2} y \cos \left(\omega_{d} t\right),
$$

and

$$
\ddot{z}(t)=-\frac{4 z}{m w_{0}^{2}} \hbar A|a(t)|^{2} e^{-2\left(z^{2}+y^{2}\right) / w_{0}^{2}} \cos ^{2} k x-\Gamma_{M} \dot{z}-2 \omega_{T}^{2} z \cos \left(\omega_{d} t\right) .
$$

Here $E$ is the input field and $k$ is the wavevector. The field is detuned by $\Delta$ from the nearest cavity resonance. The coupling between the spheres motion and the cavity field is represented by $A=\frac{\omega_{l}}{2 V_{c} \epsilon_{0}} \alpha$, where $\alpha=3 V_{s} \frac{\epsilon-1}{\epsilon+2}$ and and $V_{s}$ and $V_{c}$ are the volume of the sphere and cavity mode respectively. The square of the Paul trap frequency is given by $\omega_{T}^{2}=\frac{2 Q V_{0}}{m r_{0}^{2}}$, where $Q$ is the charge on the sphere, $V_{0}$ is the voltage amplitude applied to the trap electrodes, $m$ is the mass of the sphere and $r_{0}$ is the electrode separation. The Paul trap frequency is related to the secular frequency $\omega_{S}$ and the micromotion frequency $\omega_{d}$ by $\omega_{T}^{2}=\sqrt{2} \omega_{d} \omega_{S}$. The mechanical damping determined by the gas is given by $\Gamma_{M}$. Although not discussed here the Paul trap must be operated within a region of stability ${ }^{16}$.

Figure 3 shows the temporal dynamics of a silica sphere of radius $200 \mathrm{~nm}$ in the hybrid trap. We consider the case of one unit of elementary charge on the sphere, and an AC voltage of amplitude $600 \mathrm{~V}$ applied to the Paul 
trap electrodes at a frequency $\omega_{d}=2 \pi 1.5 \mathrm{kHz}$. In the simulations the cavity has a finesse of 15,000 and FWHM cavity linewidth of $\kappa=288 \mathrm{kHz}$. The figure shows that the particle is quickly trapped along the field direction (x-direction) where it is confined to a single antinode. The motion is largely unperturbed along the other two axes.

In our experiment we observe the light scattered by the nanosphere. Figure 4 contains plots of the calculated scattered light as the particle moves through the standing-wave pattern. Also shown are the calculated momentum and the position. This figure shows the broad features of the capture process, which in this particular case occurs at $t \simeq 0.065 \mathrm{~s}$. The scattered light is observed to increase as the particle becomes trapped about a single antinode of the cavity field. As cooling progresses the average scattered intensity increases as the particle becomes more localised on the antinode. Note that the low-frequency secular motion can be still observed in the scattered light, due to motion in the radial direction of the cavity light field. The cooling is confirmed by the slow decrease in momentum once the particle is trapped within the anti-node.

Figure 5 is a plot of the scattered light intensity, momentum and position of the particle at a much higher temporal resolution than shown in Fig. 4. Here the capture can be seen as a dip in the intensity (at $t \simeq 0.06495 \mathrm{~s}$ in Fig. 5) as the particle slowly pass over the node (top of the optical potential well) into the next antinode (bottom of well), where it is confined. Once captured the particle undergoes a relatively complicated motion which consists of a combination of harmonic motion in the optical potential $(10 \mathrm{~s} \mathrm{kHz})$ and a lower frequency motion due to the Paul trap $(1.5 \mathrm{kHz})$. We note that it is the motion induced by the Paul trap that keeps the particle away from the antinode, where cooling is inefficient. Also shown in this Fig. 5 is a scale diagram of the particle moving through the 1-D inteference pattern of the cavity field. This figure shows the relative size of the nanosphere with respect to the standing-wave field, indicating how the movement through the interference pattern will modulate the intensity of the light scattered by the particle.

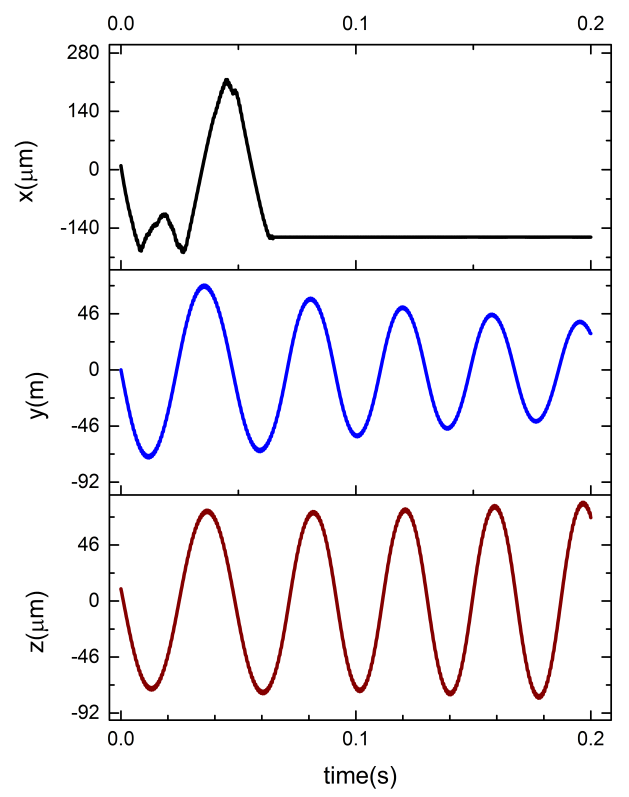

Figure 3. The calculated temporal dynamics of a silica sphere of radius $200 \mathrm{~nm}$ cooled in the hybrid trap. The particle is captured by the optical field, so that it is localised along the cavity axis (x-direction) within a well of the optical standing-wave potential. The nanoparticle is still driven in the transverse directions by the Paul trap. The simulation corresponds to $30 \mathrm{~mW}$ power input to the cavity and a laser detuning of $-\kappa / 2$ (red-detuned). 


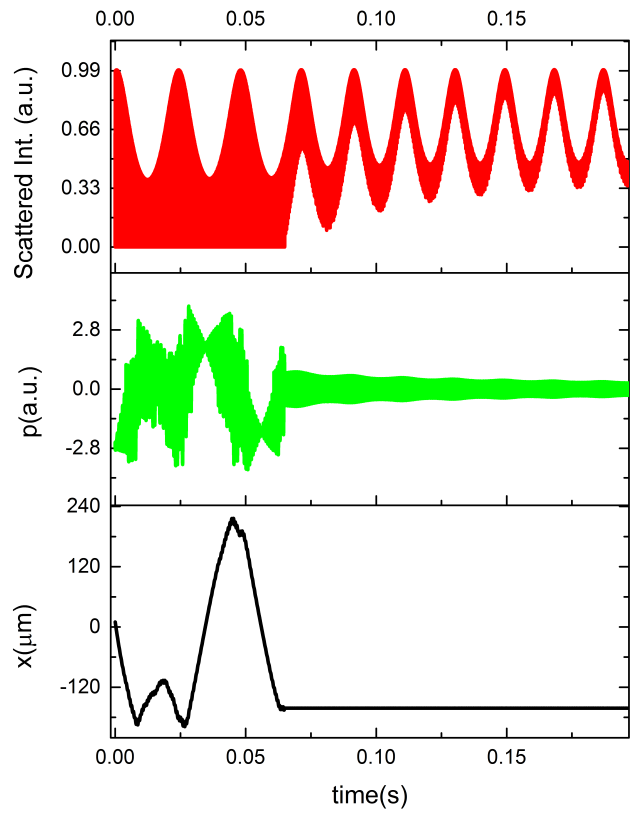

Figure 4. Calculations showing the expected light scattered by the particle as a function of time as it moves through the optical standing-wave, and is captured at $t \simeq 0.065 \mathrm{~s}$. Also shown are the calculated momentum $\mathrm{p}$ and the position $\mathrm{x}$.

\section{EXPERIMENT}

A schematic diagram of the experimental setup including the hybrid trap used for cooling the nanoparticles is shown in Fig. 6 . Here the optical cavity (finesse $\approx 15000$ ) is placed within a vacuum chamber which can currently be pumped to $10^{-6}$ mbar using a turbo molecular pump. The ion trap, which is mounted on a x-y-z translation stage, is placed within the centre of the cavity. The stage allows the particle trapped in the ion trap to be centred within the cavity field. A long working-distance microscope with camera is used to image the trap region. This camera is replaced by a amplified InGaAs diode to detect the scattered light from the particle as function of time. A single frequency (Innolight) Nd:YAG laser is used to produce the cavity field. The Pound-Drever-Hall scheme, which applies feedback to a piezoelectric actuator in the laser, is used to lock the laser onto the TEM $\mathrm{T}_{00}$ cavity resonance. The laser beam is split into two components, with a weak beam used for locking, and the other shifted in frequency using two AOMs to provide red-detuned light for cooling. The weak beam is at a low enough intensity that it does not significantly perturb the motion of the trapped particle. In all experiments the cooling beam is detuned to the red by $\kappa / 2 \approx 144 \mathrm{kHz}$. Nanospheres of radius $209.5 \mathrm{~nm}$ are introduced into the trap at a pressure of approximately $0.1 \mathrm{mbar}$ using a piezoelectric speaker that is driven by a signal generator, as shown in Fig. 1. To prevent clumping of the particles they are sonicated in a methanol solution for greater than 1 hour before being deposited to dry on the piezoelectric speaker. To prevent accumulation of the nanoparticles on the mirrors movable shields are placed in front of each mirror while the particles are loaded into the Paul trap. After $\approx 1 \mathrm{~s}$ of continuous loading, the piezoelectric speaker is turned off and the shields are removed. The weak laser beam is then locked onto resonance, and cooling occurs within the field of the stronger red-detuned beam that counter-propagates to the locking beam. The vacuum chamber is then pumped down to a low enough pressure that damping due to cavity cooling dominates over damping due to residual gas. This corresponds to a pressure of approximately $10^{-4} \mathrm{mBar}$. 

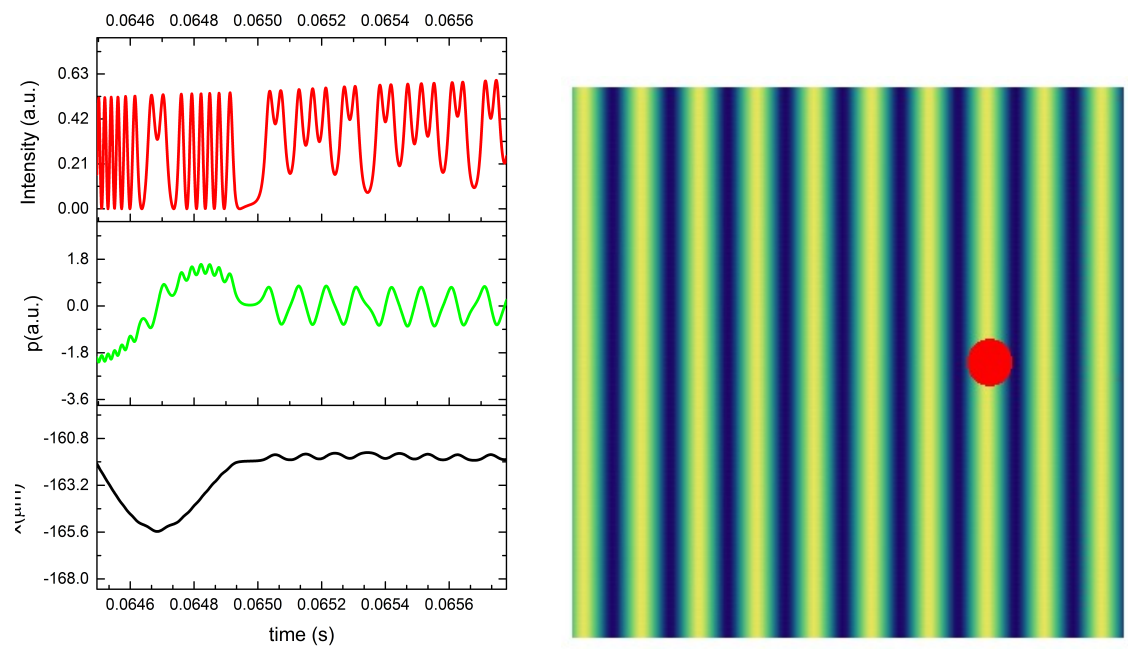

Figure 5. Calculated scattered light intensity, momentum $\mathrm{p}$ and position $\mathrm{x}$ as a function of time at a higher temporal resolution than in Fig. 4. Here the detailed dynamics after the capture of the particle in an antinode can be seen. Also shown is the size of the sphere with respect to the intracavity standing wave field.

\section{RESULTS}

Very rapidly after the cooling beam is turned on the particle is observed to localise on a single antinode, typically for durations exceeding $100 \mathrm{~ms}$. Figure 7 is a plot of the recorded intensity of the scattered light from the nanosphere within the hydrid trap as a function of time. at low time resolution. The increase in average intensity signals the capture of the particle in an antinode. When it is not captured in the antinode, it is only levitated by the Paul trap. Here the particle moves over many fringes, and the average light intensity is lower.

Figure 8 is a high temporal resolution plot of the capture and cooling process. Before the capture, a high frequency oscillation is observed. This is characteristic of the particle moving at high speed across the standingwave. The amplitude of this modulation is lower than the rest of the plot due to the reduced gain of our detector at frequencies exceeding $100 \mathrm{kHz}$. It shows the characteristic decrease in scattered intensity as the particle decelerates over the last antinode before it is captured. Once captured the average intensity increases as the particle is localised about a single antinode. Importantly, however, the amplitude of the modulation decreases with time after capture. The variation in the light scattered by the captured particle during cooling is relatively complicated, as the motion of the particle at the drive frequency and at the higher optical trapping frequency undergoes a type of FM modulation as it oscillates across the interference pattern. This produces a range of frequency components as shown in the power spectrum of Fig. 9a. This spectrum is calculated over a short interval after capture, and reveals a range of frequency components, including stronger features at twice the drive frequency $2 f_{d}$, twice the optical frequency $2 f_{\text {opt }}$ and at $f_{\text {opt }} \pm f_{d}$. Figure $9 \mathrm{~b}$ is a power spectrum of the data $10 \mathrm{~ms}$ after capture. It shows that all frequency components visible in Fig. 9a, except those due only to the drive frequency, are significantly reduced by the cavity cooling. The relative heights of these components can be used to calculate the relative energy loss over $10 \mathrm{~ms}$. For an initial temperature of $300 \mathrm{~K}$ this particle reaches a final temperature of $10 \mathrm{~K}$. 


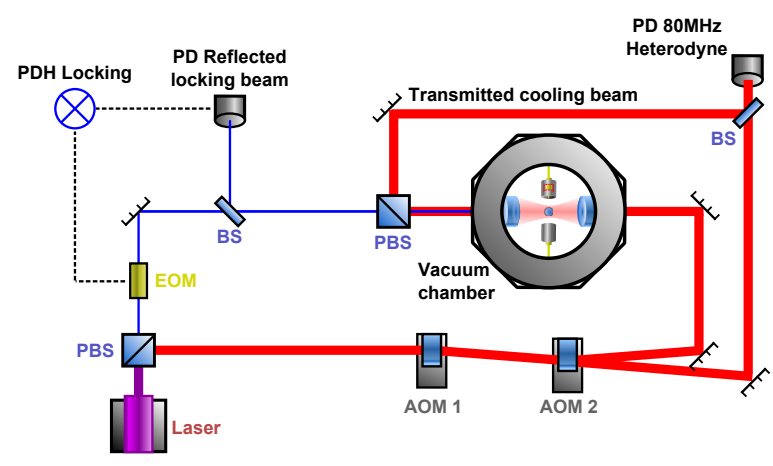

Figure 6. A schematic diagram of the experimental setup including the hybrid trap used for cooling the nanoparticles. Also shown is the optical path for the cooling and locking beams.

\section{CONCLUSIONS}

We have demonstrated cavity cooling of $200 \mathrm{~nm}$ radius nanospheres in a hybrid opto-electrical trap. Using a modest finesse cavity we show that 1-D centre-of-mass cooling to approximately $10 \mathrm{~K}$ over $10 \mathrm{~ms}$ can be achieved in vacuum. As we primarily cool along one direction of the Paul trap, noise currently limits the cooling time to at best $300 \mathrm{~ms}$. By using a higher finesse cavity we can operate in the sideband-resolved limit, with much greater cooling rates, which will allow us to approach the ground-state of the oscillator. In addition, by aligning the optical potential to have a component in each of the three axes of the Paul trap, cooling in 3-D will allow us to keep the particle captured and cooled within a single antinode indefinitely. 


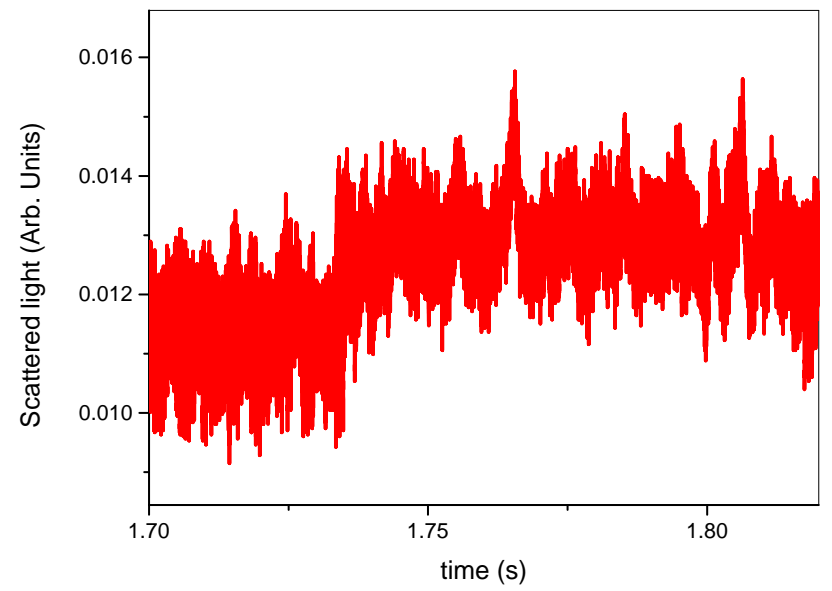

Figure 7. A low temporal resolution plot of the recorded intensity of the scattered light from a levitated nanosphere within the hydrid trap as a function of time. The increase in intensity represents localisation of the particle about an antinode, and subsequent cooling.

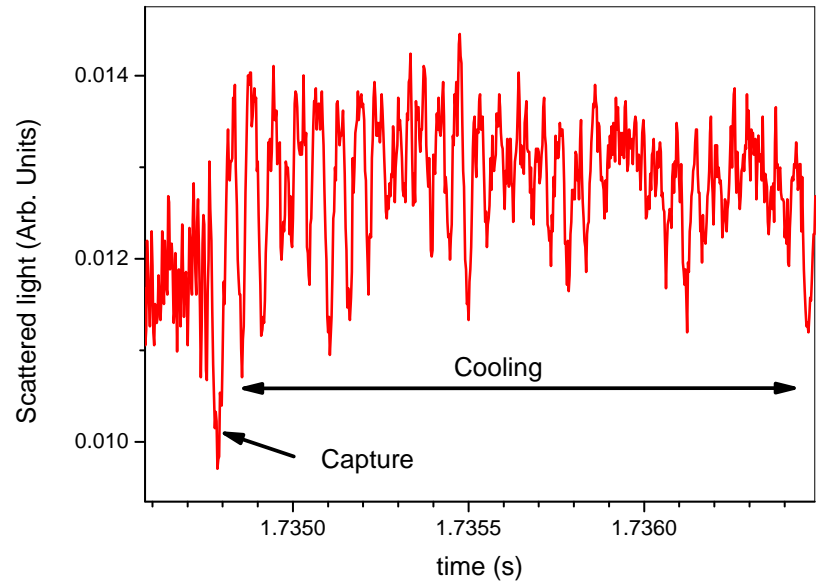

Figure 8. A high temporal resolution plot in the region where the nanosphere is initially captured about an antinode. Cooling is evidenced by the continual decrease in amplitude of the oscillation after capture. 


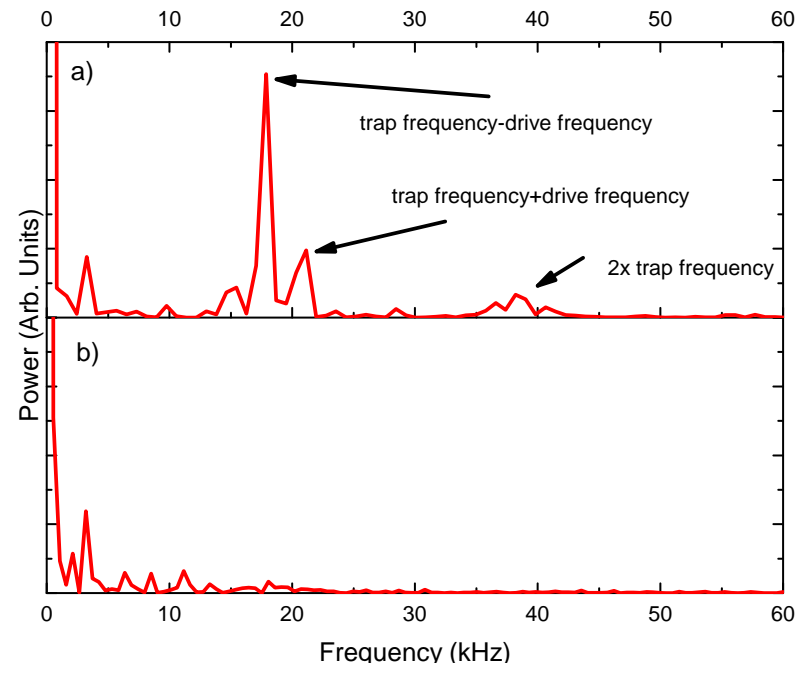

Figure 9. Power spectra calculated from the the scattered light. a) Spectrum from just after capture and b) 10 millisconds after this time. 


\section{REFERENCES}

[1] O. Romero-Isart et al., Phys. Rev. Lett. 107, 020405, (2011).

[2] M. Bahrami, M. Paternostro, A. Bassi, and H. Ulbricht, Phys. Rev. Lett. 112, 210404 (2014).

[3] A. A. Geraci, S. B. Papp, and J. Kitching, Phys. Rev. Lett. 105, 101101 (2010).

[4] J. D. Thompson et al., Nature 452, 72 (2008); A.M. Jayich et al., New J. Phys 10, 095008 (2008).

[5] P. Horak et al., Phys. Rev. Lett. 79, 4974 (1997).

[6] P. Asenbaum et al., Nature Commum. 4, 2743 (2013).

[7] N. Kiesel et al., Proc. Natl Acad. Sci. USA 110, 14180 (2013)

[8] J. Millen, T. Deesuwan, P. F. Barker, and J. Anders, Nature Nano. 9, 425 (2014).

[9] J. Gieseler, R. Quidant, C. Dellago, and L. Novotny, Nature Nano. 9, 358 (2014).

[10] O. Romero-Isart, M. L. Juan, R. Quidant and J. I. Cirac, New J. Phys 12, 033015 (2010).

[11] D. E. Chang et al., Proc. Natl. Acad. Sci. USA 107, 1005 (2010).

[12] P. F. Barker and M. N. Shneider, Phys. Rev. A 81, 023826 (2010).

[13] G. A. T. Pender et al., Phys. Rev. A 85, 021802 (2012).

[14] T. S. Monteiro et al., New J. Phys. 15, 015001 (2013).

[15] J. Gieseler, B. Deutsch, R. Quidant, and L. Novotny, Phys. Rev. Lett. 109, 103603 (2012).

[16] J. Millen et al., arXiv:1407.3595 (2014). 